\title{
SISTEMA CONSTRUCTIVO «QUINCHA PREFABRICADA»
}

\author{
(«PREFABRICATED CANE LATHING» CONSTRUCTION SYSTEM)
}

Anibal Díaz Gutiérrez, Ingeniero Director de Normalización

I.N.I.N.V.I. - PERU

\section{RESUMEN}

La "Quincha Prefabricada» consiste básicamente en el empleo de bastidores de madera aserrada, rellenados con cañizo redondo, caña brava, tiras de bambú, todos ellos colocados en el bastidor en forma trenzada para su autofijación sin necesidad de usar clavos; estos paneles, después de ser montados y fijados en sitio constituyendo paredes, son revocados con barro mezclado con paja, formando una primera capa; $y$, finalmente, reciben una última capa de revoque utilizándose materiales como el barro, cemento, yeso u otros, dependiendo de las preferencias, costos, de las condiciones climatológicas $u$ otras a las que estará expuesta la pared o de las preferencias del usuario.

Como techado se emplea un techo liviano a base de una estructura portante de madera y cobertura de caña y torta de barro con paja. Para zonas de alta precipitación pluvial se fija, sobre la torta de barro, planchas de asbesto-cemento, planchas de zinc $u$ otras.

\section{SUMMARY}

"Prefabricated cane lathing» basically involves the use of frames of sawn timber, filled with round wattle, cane and strips of bamboo, all plaited together so that they can be placed and fixed in the frame wihout nails being required. Once these panels have been assembled and fixed in place to provide walls, they are covered with a mixture of mud and straw forming a primary layer, and finally, they are given a top layer using such materials as mud, cement, plaster and other substances, depending on preferences, costs and climatic or other conditions to which the wall is to be exposed, or on the preferences of the user.

A light roof is used for roofing, based on a wooden support structure and a covering made of cane and a mud cast packed with straw. For regions of high rainfall, planks of asbestos and cement, zinc or other materials are fixed over the mud cast.

\section{PRESENTACION}

En el Perú, hace ya varios años que el Instituto Nacional de Investigación y Normalización de la Vivienda - ININVI viene investigando, como una parte importante de su quehacer, las denominadas Tecnologias Tradicionales -adobe, tapial, quincha, madera- con el propósito de recuperarlas y mejorarlas.

Mediante la utilización de las tecnologias tradicionales más del $60 \%$ de nuestra población ha resuelto de alguna manera - muchas veces insatisfactoria - sus necesidades de habitación. Sin embargo, utilizando los mismos materiales tradicionales es posible lograr soluciones con adecuadas condiciones de habitabilidad, durabilidad y seguridad.

En esta oportunidad, presentamos un Sistema tradicional mejorado que estamos denominando "Quincha Prefabricada", con lo cual creemos estar aportando una solución válida para el problema habitacional y respetando a la vez las caracteristicas peculiares de la quincha, que constituyen, sin ninguna duda, una expresión cultural propia de nuestro pueblo, tal como puede apreciarse en diversos monumentos arquitectónicos, asi como en humildes viviendas.
El Sistema de "Quincha Prefabricada" que ha quedado registrado como Sistema Constructivo No Convencional de acuerdo a la R.D. N. ${ }^{\circ}$ 001-84VC-9602, y por lo tanto puede ser utilizado libremente en todo el pais, es un sistema sumamente versátil puesto que puede utilizarse tanto de manera masiva, mediante sencillos procesos de industrialización, que permiten prefabricar los paneles que requieren las viviendas, como en la autofabricación y autoconstrucción, empleando herramientas de carpintería y albañileria elementales.

Cabe señalar que de acuerdo a los resultados experimentales obtenidos, el Sistema presenta muy buenas caracteristicas de aislamiento térmico y acústico y de otro lado, ha mostrado un excelente comportamiento frente a solicitaciones sísmicas; además, su costo se mantiene muy por debajo de los sistemas constructivos convencionales en aquellos lugares donde existe facilidad de obtener madera y caña que constituyen los insumos principales del Sistema. Por ello, consideramos que, adecuadamente utilizado, el Sistema de "Quincha Prefabricada" puede constituir una excelente solución habitacional.

Para la elaboración del documento técnico que presentamos, nos hemos basado no sólo en las pruebas hechas en el Laboratorio sino en expe- 
riencias constructivas concretas realizadas mediante la construcción de Núcleos Básicos de Vivienda, uno en el propio local de nuestra Institución y otro en la ciudad de Popayán de Colombia que ha sufrido un severo movimiento sísmico. Por ello, es que la información que proporcionamos tiene contenido tanto teórico como práctico, siendo esta última característica la que nos mueve a darlo a conocer esperando que pueda ser acogido por otros paises iberoamericanos, cuyas realidades socio-económicas son muy similares a las del Perú.

\subsection{GENERALIDADES}

La "Quincha" es un sistema constructivo tradicional, que viene usándose en el Perú desde épocas pre-hispánicas y que fue empleada profusamente en la época colonial habiendo llegado a su máximo desarrollo en el siglo XVIII. Quedan aún muchos monumentos históricos en el pais, construidos en base a quincha, así como viviendas cuya antigüedad sobrepasa el siglo y que han resistido la acción del tiempo, del clima y de movimientos sísmicos severos.

La presencia actual de la quincha en la construcción de viviendas campesinas se puede apreciar prácticamente a todo lo largo de la costa peruana.

\subsection{DESCRIPCION}

\subsection{MATERIALES USADOS}

Los materiales básicos constitutivos de la quincha son: la madera, la caña y los materiales de revoque.

\subsection{La madera}

Es el material que reúne más ventajas que ningún otro conocido actualmente. Es el recurso renovable por excelencia, se encuentra en grandes cantidades y repartida en casi todo el mundo; sus reservas se renuevan indefinidamente. Cuando se usa en forma correcta, dura indefinidamente.

Su elasticidad le permite absorber los esfuerzos que causarian la fractura de otro material.

Se puede manufacturar con herramientas muy sencillas.

Es el único material con el que se puede construir totalmente una vivienda, desde la estructura, pisos y paredes hasta los muebles.

Según su anatomia, las células que constituyen la madera o tejido leñoso, son diferentes en forma y funciones tratándose de coniferas o latifolladas.
Los bosques de coniferas se desarrollan principalmente en las zonas frías y templadas del hemisferio norte.

En las zonas tropicales de Africa, América, Asia y Oceania, crecen bosques integrados por una gran variedad de especies latifoliadas.

En el Perú y en general en Latinoamérica, la madera más abundante es la latifoliada.

\subsubsection{Secado}

El secado

El secado de la madera puede hacerse natural o artificialmente.

El contenido de humedad de la madera para ser empleada en construcción, debe estar comprendida entre $14 \%$ y $20 \%$.

\subsubsection{Preservación}

La madera es susceptible de ser atacada por agentes biológicos y no biológicos, que disminuyen su calidad. Hay maderas que tienen resistencia natural al ataque, pero éstas son las menos.

A fin de darle durabilidad a la madera frente al ataque de los hongos e insectos xilófagos, se procede a su preservación mediante compuestos químicos.

La aplicación de estos preservantes, puede efectuarse por varios métodos entre los que se pueden citar:

Tratamiento sin presión:
a) Brocha
b) Pulverización
c) Inmersión
d) Baño caliente o frío

Tratamientos con presión:

a) A célula llena

b) A célula vacia

\subsubsection{Clasificación}

La Junta del Acuerdo de Cartagena - Proyecto PADT - REFORT, ha estudiado 20 especies maderables latifolladas peruanas, adecuadas para ser empleadas en construcción, agrupándolas estructuralmente en tres, en función de su resistencia y densidad básica.

- El Grupo A, que comprende a las maderas de mayor resistencia, cuyas densidades están por lo general en el rango de 0,90 a $0,71 \mathrm{~g} / \mathrm{cm} 3$. 
- El Grupo B, que comprende a las maderas de mediana resistencia y sus densidades se sitúan entre el rango de 0,70 a $0,56 \mathrm{~g} / \mathrm{cm} 3$.

- El Grupo C, que comprende a las maderas de menor resistencia, sus densidades se sitúan en el rango de 0,55 a $0,40 \mathrm{~g} / \mathrm{cm} 3$.

\subsection{LA CANA}

Para el relleno de los bastidores de madera de los paneles, se utilizaba "caña" palabra que engloba muchas especies de bambúes. Generalmente las más recomendadas para ser utilizadas en paneles de quincha son las siguientes especies y géneros:

\section{Nombre Cientifico}

a) Chusquea spp.

b) Gynesium sagittatum

c) Guadua angustifolia

d) Género de bambusas

e) Genero de arundinarias

f) Género de dendrocálamus

g) Género de gigantochloas

De toda esta variedad de cañas, las que más ventajosamente pueden ser empleadas en el sistema de "QUINCHA PREFABRICADA" son:

\subsubsection{El Carrizo}

Es una caña hueca, de sección transversal redonda o casi redonda y con tabiques transversales rígidos, estratégicamente colocados, que evitan la ruptura de la caña al curvarse.

El carrizo es bastante resistente. Tiras de carrizo sometidas a ensayo de tracción, han logrado resistir cargas por encima de $1.000 \mathrm{~kg} / \mathrm{cm} 2$.

Su diámetro puede variar de 1/2" a 1 1/2", pudiendo alcanzar 6 o más metros de altura.

El carrizo es una gramínea que se desarrolla fácilmente en las zonas cálidas y donde abunde agua superficial.

\subsubsection{La Caña Brava}

Es una graminea de tallo casi sólido, con las hojas fuertemente adheridas al tallo, formando un conjunto bastante denso. Al igual que el carrizo se desarrolla con mucha facilidad en las playas $y$ riberas de los ríos costeros y de ceja de selva.

Se reconoce que la caña brava ha llegado a la madurez cuando aparece la florescencia.
Para la preparación de los paneles de quincha debe ser preferido al carrizo, por su mayor durabilidad.

\subsubsection{El Bambú}

Es una caña, la de mayor desarrollo en la familia de las gramíneas, que crece entre $40 \mathrm{~cm}$ y $80 \mathrm{~cm}$ diarios y puede alcanzar una altura de $40 \mathrm{~m}$ en menos de dos meses.

Hay más de 600 especies de bambúes. Todos tienen tallos lisos, generalmente huecos, articulados con un fuerte tabique transversal obturante en cada articulación.

En proporción a su peso, las cañas de bambú son muy fuertes.

Puede ser utilizado como material en construcciones, 3 o 4 años luego de ser plantado.

Los bambúes son plantas de los trópicos y de las regiones cálido-templadas y son muy ávidos de agua.

Algunas especies llegan a alcanzar un grueso de más de $30 \mathrm{~cm}$ de diámetro.

\subsection{EL PANEL}

Consiste básicamente en un bastidor de madera estructural aserrada que constituye la estructura del mismo y que en el caso del panel típico, está conformado por 2 parantes, 4 travesaños y 4 semidiagonales a fin de darle cierto grado de indeformabilidad.

La mayoría de las piezas de madera del bastidor son de $30 \mathrm{~mm}$ x $65 \mathrm{~mm}$ (1 1/2" x 3"') y de $20 \mathrm{~mm}$ x $30 \mathrm{~mm}(1 " \times 11 / 2 ")$ de escuadria.

Siendo el sistema de Quincha Prefabricada, modular y racionalizado, se reduce al máximo la variedad de paneles.

Todos los paneles tienen $2,40 \mathrm{~m}$ de alto $\mathrm{y}$ dos opciones de ancho, $0,60 \mathrm{~m}$ y $1,20 \mathrm{~m}$.

Se dispone de la siguiente variedad de paneles:

Panel Típico (Fig. 1 en la pág. siguiente)

Medio Panel

Panel Puerta 1

Panel Puerta 2

Panel Ventana Baja

Panel Ventana Alta

Panel Tímpano Rectangular

Con los cuales se puede edificar una vivienda. 


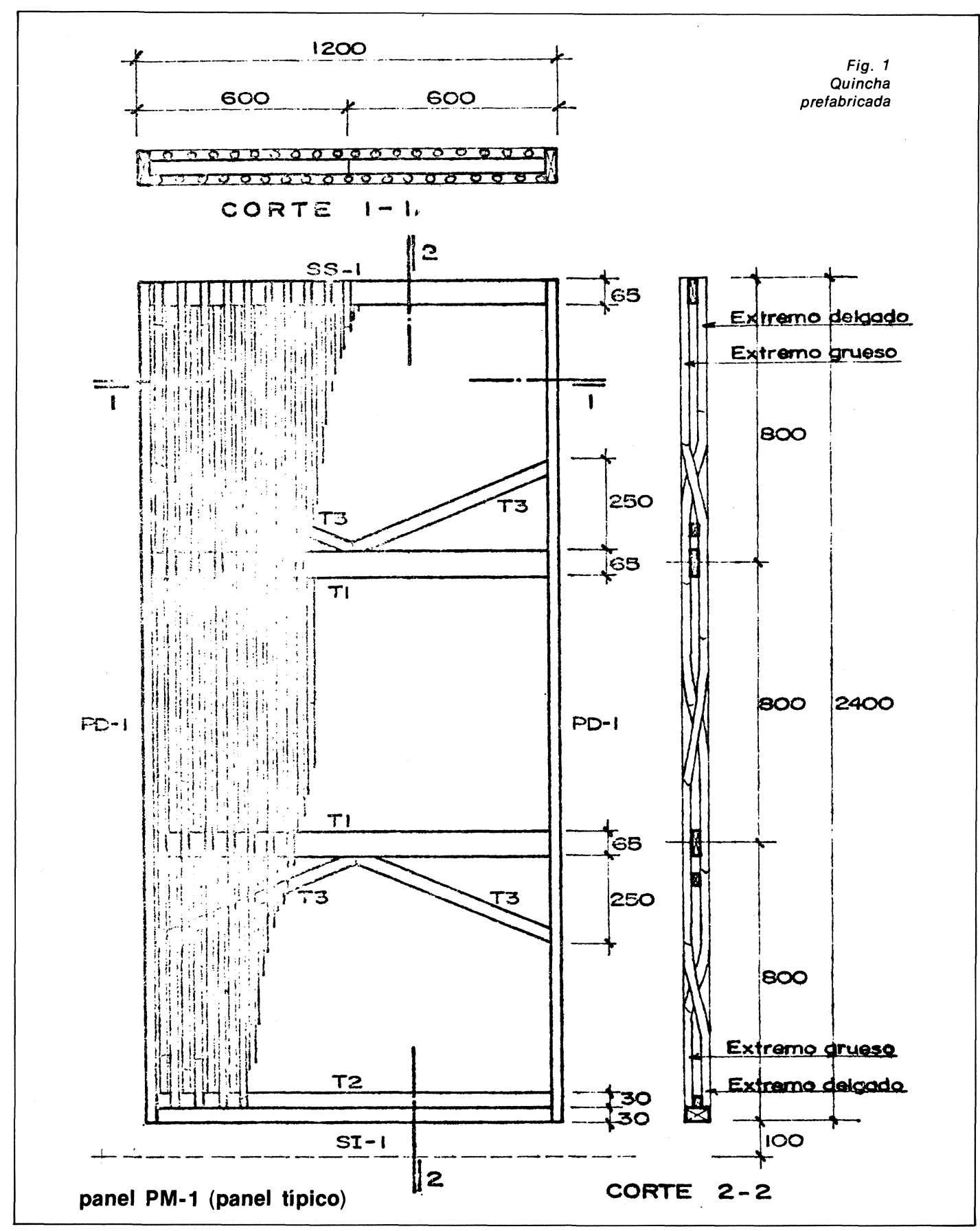

\subsection{ARMADO DE PANELES}

En su confección, se emplea madera estructural seca, con un contenido de humedad inferior a $20 \%$ y todas las uniones a tope utilizándose clavos.

Las secciones de las piezas de madera deben ser uniformes, siendo preferible que las superficies sean cepilladas.

Es importante que tanto el alto, como el ancho de los paneles tengan medidas precisas, acercándose a perfectos rectángulos.
Ayuda a conseguirlo si en el proceso de prefabricación, se cortan en una sola operación, las piezas de madera que deban tener la misma longitud.

Si la madera estructural que se usa, tiene tendencia a rajarse en sus extremos cuando es clavada, se recomienda pretaladrar las piezas, utilizando una broca que produzca una perforación del orden del $80 \%$ del diámetro del clavo a ser empleado.

\subsection{EL RELLENAdo de CAÑA DEL PANEL}

El carrizo y la caña brava, deben ser empleadas en rollizo y sin pelar para una mejor adherencia 
del revoque; y con un diámetro comprendido entre 0/1/2" y 0/3/4". Los bambués y cañas de otros géneros de gran diámetro, deben utilizarse partidas en tiras longitudinales de 1" de ancho. EI espesor de estas tiras, no debe ser menor de $1 / 2$ ", ni mayor de $3 / 4$ ".

Una vez preparada la estructura de los paneles, se procede a su relleno mediante el trenzado de las cañas, alternándose los extremos delgados con los gruesos para obtener anchos iguales en los dos extremos del panel.

Si se utilizan tiras, deberá alternarse la cara lisa de una tira, con la cara pulposa de la tira vecina.

Las cañas o tiras deben quedar en toda su longitud fuertemente presionadas unas con otras para proporcionar mayor rigidez al panel.

Se recortan las cañas después que se haya terminado de rellenar el panel o de lo contrario se cortan las cañas a una misma longitud previamente determinada, antes de proceder al trenzado; esta última modalidad es preferible.

\subsection{REVOQUES}

El montaje de los paneles prefabricados de quincha se realiza in situ sin revocar; de lo contrario su manipuleo sería dificultoso. (Un panel tipico de $1,20 \mathrm{~m}$ de ancho sin revocar pesa aproximadamente $50 \mathrm{~kg}$ y revocado pesa $350 \mathrm{~kg}$ aproximadamente).

Se pueden utilizar varios tipos de revoques en base a diferentes materiales. Desde los más económicos como el barro mezclado con paja, hasta los más sofisticados. No debe descuidarse factores tan importantes como adherencia, resistencia al desgaste, aislamiento térmico y acústico, resistencia al impacto, menor fisuración por baja contracción de secado o fraguado, durabilidad y otros factores de habitabilidad.

Se recomiendan los siguientes tipos de revoques de los muros de quincha:

Tipo 1:

- Un revestimiento primario de barro y paja en una proporción de $2,0 \mathrm{~kg}$ de paja por cada $100 \mathrm{~kg}$ de tierra, enrasado con el marco del panel.

- Un revestimiento secundario aplicado sobre el revestimiento primario cuando éste se haya secado completamente y de $1,5 \mathrm{~cm}$ a $2,0 \mathrm{~cm}$ de espesor, de barro preparado con tierra arenoarcillosa cernida.

Esta mezcla debe rellenar todas las fisuras del revestimiento primario.
Tipo 2:

- El revestimiento primario y secundario igual que el Tipo 1.

- Un revestimiento final de "lechada" de cemento aplicada con brocha a modo de pintura.

La superficie usualmente debe pintarse dos veces.

La consistencia de la pintura para la primera mano deberá ser como la de la leche y para la segunda, como la de una crema espesa.

La capa final debe ser aplicada sobre la pared seca en su interior y húmeda en su superficie; para lo cual se procede a su humedecimiento inmediatamente antes del pintado.

Después de 24 horas de la primera mano, se aplica la segunda, previo humedecido de la superficie.

El curado de la superficie es de mucha importancia, recomendándose iniciarlo 12 horas después de la segunda mano, mediante humedecimiento, continuándose este proceso tres veces diarias durante un periodo de 2 a 3 dias.

Tipo 3:

- El revestimiento primario igual que el del revoque Tipo 1.

- Un estucado final de yeso de $1,5 \mathrm{~cm}$ a $2,0 \mathrm{~cm}$ de espesor.

Tipo 4:

- El revestimiento primario igual que el del revoque Tipo 1.

- Un revestimiento final de $1,5 \mathrm{~cm}$ a $2,0 \mathrm{~cm}$ de una mezcla de yeso-cemento (diablo) en la proporción en volumen $4: 1$ y agua.

Tipo 5:

- Un revestimiento primario de suelo-cemento enrasado con el marco de los paneles.

Deberá preferirse utilizar tierra arenosa y el porcentaje de cemento a emplear dependerá del tipo de tierra, pero usualmente puede variar de $7 \%$ a $10 \%$ en volumen.

Este revestimiento primario deberá ser curado por lo menos durante una semana mediante humedecimiento periódico. 
- Un revestimiento final de $1,5 \mathrm{~cm}$ a $2,0 \mathrm{~cm}$ de mortero cemento-cal-arena en la proporción en volumen 1:1:5.

\section{Tipo 6:}

- Un salpicado inicial con mortero cemento-calarena 1: 1:5 que cubra parcialmente todas las superficies de caña.

- Un revoque final aplicado a las superficies, a las 24 horas después del salpicado inicial y con el mismo mortero 1:1:5.

\section{0: ASPECTOS ARQUITECTONICOS}

El Sistema de Quincha Prefabricada tiene carácter modular siendo el módulo básico el componente panel; es necesario que el diseño arquitectónico de la vivienda tenga en cuenta este módulo.

Todos los ambientes en planta, deben tener como longitud interior libre, un múltiplo de $0,60 \mathrm{~m}$ (medio panel).

Los vanos de puertas de $0,87 \mathrm{~m}$ de ancho, por $2,00 \mathrm{~m}$ de alto, 0 de $0,62 \mathrm{~m}$ de ancho por $2,00 \mathrm{~m}$ de alto.

El nivel de piso terminado debe tener una cota minima de $+0,10 \mathrm{~m}$ tomando como referencia la cota $\pm 0,00$, para la vereda frontal o perimetral; 0 para el terreno natural circundante.

El espesor de los muros terminados deben ser de $0,10 \mathrm{~m}$ como mínimo.

Se recomienda techados de una o dos aguas. La pendiente es función de la intensidad de la precipitación pluvial predominante de la zona, así como de la rugosidad del material de cobertura. Pero en general esta pendiente puede variar de $15 \%$ a $30 \%$.

La longitud de los aleros perimetrales de protección son definidos también por la intensidad de la precipitación pluvial predominante; pero en todo caso, deben ser lo suficientemente largos como para asegurar que los muros no sean humedecidos por el agua de lluvia que cae directamente o por salpicadura.

Las edificaciones de "Quincha Prefabricada" deben en lo posible ser ubicadas alejadas del perimetro del lote para facilitar el revoque de todas las superficies y además eliminar la necesidad de construir muros cortafuego.

\subsection{PROCEDIMIENTO CONSTRUCTIVO}

\subsection{PREPARACION DEL TERRENO}

Se elimina la capa superficial de terreno natural que contiene material orgánico y cuyo espesor varía de $30 \mathrm{~cm}$ a $40 \mathrm{~cm}$.

\subsection{TRAZADO Y EXCAVACION}

Una vez eliminada la capa superficial en toda el área que ocupará la edificación, se nivela y se procede al trazado en forma convencional, utilizándose puentes 0 balizas de madera y fijándose ejes de muros y anchos de cimientos y sobrecimientos.

Dimensiones de las zanjas:

Ancho: $\quad 30 \mathrm{~cm}$, a lo más $40 \mathrm{~cm}$.

Profundidad: $40 \mathrm{~cm}$, mínimo.

\subsection{VACIADO DEL FONDO DEL CIMIENTO}

Con una mezcla pobre de cemento-árido de rio en la proporción 1:12 en volumen, se hace el vaciado sobre el fondo de las zanjas de una capa de aproximadamente $10 \mathrm{~cm}$ de espesor.

\subsection{PREPARACION DE COLUMNAS DE MADERA}

Con madera estructural seca, se preparan columnas de $65 \mathrm{~mm} \times 65 \mathrm{~mm}$ (3" $\times 3$ 3") de sección y de $3 \mathrm{~m}$ de largo. Es necesario preservar las columnas, mediante el método adecuado de preservación citados en 3.1.2.

Se aplica a cada columna en toda su superficie, dos manos de pintura impermeabilizante o emulsión asfáltica, la que normalmente demora en secarse 24 horas o más.

La impermeabilización debe ser hecha con más cuidado, de las superficies de madera que quedarán en contacto con el hormigón de la cimentación.

Para una mejor fijación de la columna a la cimentación, donde irá empotrada verticalmente, en uno de sus extremos y en $50 \mathrm{~cm}$ de su longitud, se introducen 8 clavos de 4", dos por cada cara (únicamente la mitad de sus longitudes), después de lo cual se les dobla $45^{\circ}$ con el eje longitudinal de la columna.

\subsection{UBICACION DE LAS COLUMNAS}

Estas irán ubicadas en la edificación de quincha en los siguientes puntos: 
a) En las esquinas

b) En los encuentros en $\mathrm{T}$ de muros

c) En los encuentros en + de muros

d) En los puntos terminales de muros

e) Intercalados en los muros a intérvalos no mayores de $3,60 \mathrm{~m}$.

(3 paneles típicos de $1,20 \mathrm{~m}$ de largo unidos colinealmente).

Las longitudes de las columnas deben ser tales que sobresalgan 5 o $10 \mathrm{~cm}$ sobre los paneles, cuando estos se monten en obra; al final se recortará el exceso de longitud de las columnas para que queden exactamente a la misma altura de los paneles.

\subsection{VIGA DE CIMENTACION}

Las edificaciones de quincha por ser livianas no necesitan viga de cimentación, a excepción que la capacidad portante del suelo sea inferior a 0,5 $\mathrm{Kg} / \mathrm{cm} 2$. En este caso, se coloca una viga de hormigón armado $403 / 8 "$, de $20 \mathrm{~cm} \times 20 \mathrm{~cm}$ continua integrada a la parte superior del cimiento.

\subsection{MONTAJE DE LAS COLUMNAS DE MADERA}

Son colocadas perfectamente verticales y apoyadas en el fondo del cimiento de hormigón pobre.

Si el cimiento lleva viga de cimentación, las columnas deben quedar introducidas entre los hierros longitudinales.

\subsection{PREPARACION Y MONTAJE DE ELEMEN- TOS DE ANClAJE DE PANELES A LA Cl- MENTACION}

Para este efecto como una de las alternativas, se usan fijadores de alambre galvanizado o alambre negro 8. Con un segmento de $1,70 \mathrm{~m}$, se forma una $U$, con una separación de $7 \mathrm{~cm}$ entre las ramas y se le introduce verticalmente hasta el fondo del cimiento.

Cada panel de $1,20 \mathrm{~m}$ de ancho, debe ser fijado mediante 3 de estos elementos y el panel de 0,60 metros, mediante 2 .

\subsection{VACIADO DEL CIMIENTO}

Montados los fijadores, las columnas de madera y cualquier otro elemento a ser empotrado, se echa el cimiento convencionalmente con hormigón 1:8 o 1:10. La superficie superior debe quedar nivelada y su cota debe coincidir con la cota inferior del futuro falso piso.

\subsection{VACIADO DEL SOBRECIMIENTO}

Haciendo uso del cimiento como apoyo, se encofra el sobrecimiento de $10 \mathrm{~cm}$ de ancho por $20 \mathrm{~cm}$ de alto.

Se echa el sobrecimiento con hormigón cementoárido de río en la proporción 1:8 y con un tamaño máximo de la piedra de 1".

Se debe tener cuidado que los fijadores de alambre queden empotrados en el sobrecimiento, centrados en él y distanciadas ambas ramas $7 \mathrm{~cm}$.

La superficie superior debe quedar nivelada, sin que sobresalgan puntas de piedra. El desencofrado se efectúa a las 24 horas o de preferencia a las 48 horas.

\subsection{PREPARACION DEL SUELO PARA RECIBIR EL PISO}

El suelo debe ser recompuesto hasta un espesor de $20 \mathrm{~cm}$, con un material de granulometría apropiada y compactado a máxima densidad seca.

Si existe peligro de ascensión de humedad al piso por capilaridad, es necesario colocar sobre el suelo compactado previo al vaciado del piso, láminas de polietileno de un espesor mínimo de $0,25 \mathrm{~mm}$, con traslapes de $20 \mathrm{~cm}$ en sus bordes.

Como alternativa a la utilización de las láminas de polietileno, se puede colocar sobre el suelo compactado una capa de empedrado utilizándose piedras de $15 \mathrm{~cm}$ a $20 \mathrm{~cm}$ y rellenando los intersticios con piedra menuda. Sobre este empedrado se echa el hormigón del piso.

\subsection{VACIADO DEL FALSO PISO}

Una vez montada la instalación sanitaria y cualquier otro elemento que deba quedar empotrado en el falso piso, se echa con hormigón 1:10 (cemento-árido de río).

La cota del piso terminado debe quedar como minimo $10 \mathrm{~cm}$ por encima de la cota de la acera frontal de la vivienda o del terreno natural circundante.

\subsection{MONTAJE DE LOS PANELES}

Los paneles se montan en sitio según el plano de distribución de paneles previamente preparado. 


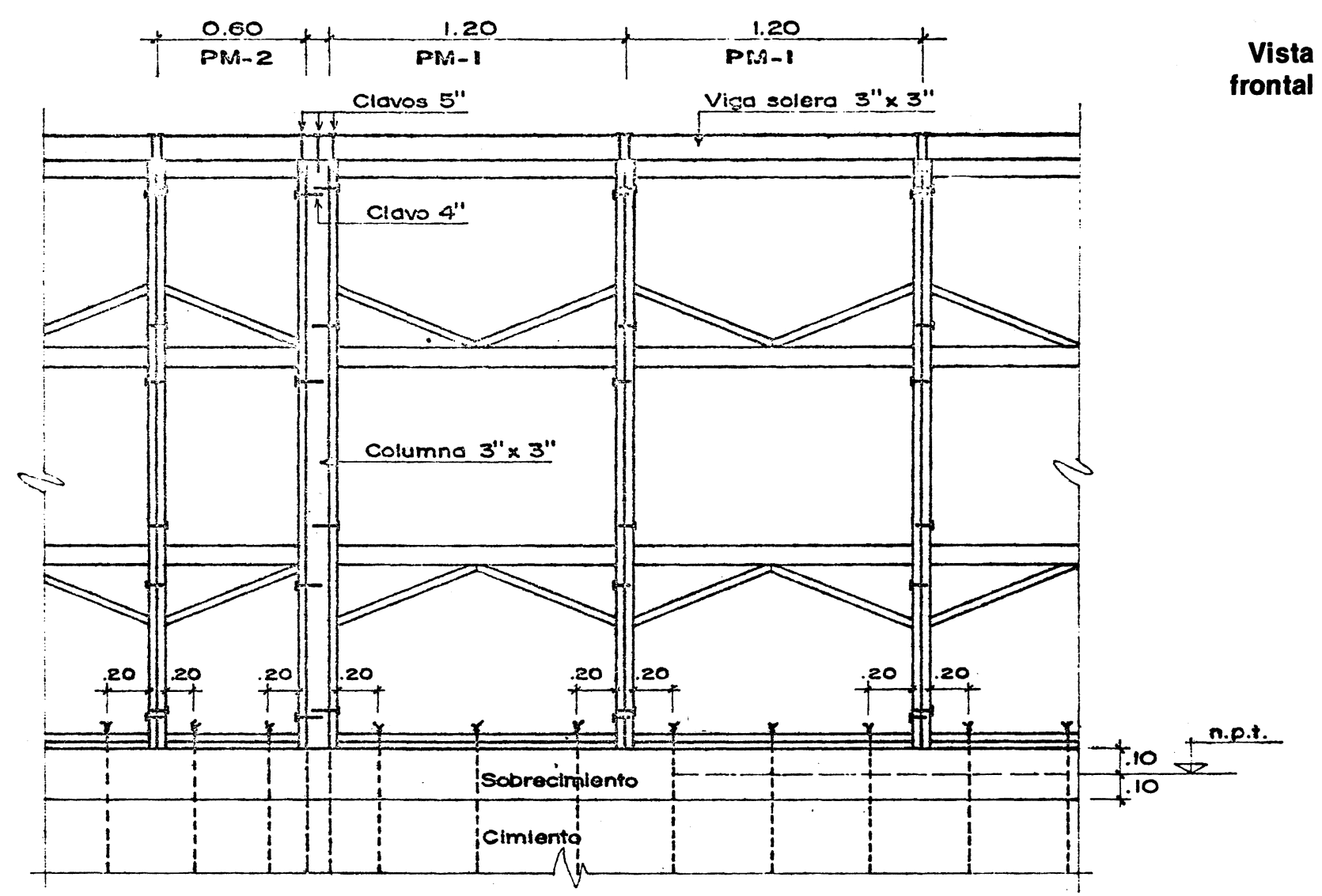

Fig. 2. - Fijación paneles entre si, a columnas, a cimentación y a viga solera.

Antes de esta operación, se aplica pintura asfáltica a la superficie superior del sobrecimiento y travesaño inferior de los paneles.

Los paneles montados, deben quedar ajustados entre columnas y fijados entre si, mediante 8 clavos de acero de 4" (4 a cada lado), que unirán los soportes de dos paneles colineales.

La unión del panel con la columna, se efectúa también mediante 4 clavos de acero de 4". (Figura 2).

\subsection{MONTAJE DE LA VIGA SOLERA}

Para la viga solera, se elige piezas de madera estructural de $65 \mathrm{~mm} \times 65 \mathrm{~mm}$ (3" x 3") de escuadria, para espaciamiento máximo de $3,60 \mathrm{~m}$ entre columnas. Para mayor espaciamiento, la sección debe ser determinada previo diseño estructural.

Antes de la fijación de la viga solera, se procede al recorte del exceso de longitud de las columnas. La viga solera se fija mediante clavos de 5" a las columnas y soportes de los paneles.

La unión a media madera de dos piezas colineales de la viga so!era, no debe coincidir con un encuentro en $T$ o en + de la misma.

\subsection{FIJACION DE LOS PANELES A LA CIMENTACION}

Una vez fijados los paneles entre sí, asi como la viga solera sobre ellos, se procede a fijar cada panel a la cimentación utilizando las tiras de alambre que han quedado empotrados en el cimiento y el sobrecimiento. La fijación se ejecuta mediante atado, previamente a lo cual es necesario verificar el alineamiento de los paneles.

\subsection{PREPARACION Y MONTAJE DE LOS TIMPANOS RECTANGULARES}

Para darle pendiente al techo, para la evacuación de las aguas pluviales, se aumenta la altura de algunas paredes de la edificación, mediante paneles prefabricados rectangulares y rellenos con caña trenzada.

La fijación de estos paneles a la viga solera, se efectúa mediante clavos de 4". Los soportes de estos paneles, deben ser continuaciones tanto en ubicación como en escuadría de los soportes de los paneles y columnas de las paredes. 


\subsection{PREPARACION Y MONTAJE DE LAS VIGUETAS INCLINADAS DEL TECHO}

Se deben elegir cuartones de madera estructural, de sección cuadrada aserrada y cepillada.

La escuadria de las viguetas debe ser determinado mediante cálculo estructural, teniendo en cuenta las cargas del techo y la resistencia de la madera elegida.

Las viguetas son montadas y fijadas a los elementos de apoyo en sus extremos mediante dos clavos de acero de 5" por apoyo. Para un mejor asiento, se practica en sus extremos rebajos en forma de $L$.

La separación centro a centro de viguetas debe ser $0,60 \mathrm{~m}$.

Con la finalidad de proporcionar aleros perimetrales de protección a la edificación, se prolongan las viguetas $0,60 \mathrm{~m}$ como mínimo del plano exterior de los paneles.

En lugar de viguetas inclinadas, se puede emplear armaduras de madera.

Una vez montadas las viguetas, se procede a completar en sitio la estructura y relleno de los tímpanos triangulares.

\subsection{PREPARACION Y COLOCACION DE LA ESTERILLA DE CAÑA EN EL TECHO}

La finalidad de esta esterilla es la de servir de soporte a la torta de barro que se coloca posteriormente; asi como posibilitar el enlucido interior del cielo raso inclinado. La esterilla puede estar constituida por bambú chancado, carrizo o caña brava 01 ".

La esterilla es fijada sobre las viguetas mediante clavos de 2 1/2" cuando se trata de bambú chancado o mediante alambre negro \# 16 o clavos.

Para darle protección lateral a la edificación, se puede prolongar la esterilla hacia el exterior.

\subsection{INSTALACION ELECTRICA}

La instalación eléctrica es convencional, recomendándose correr las conducciones sobre la esterilla de caña del techo. Las bajadas hacia los puntos de iluminación, interruptores y tomacorrientes se deben practicar verticalmente, efectuándose pequeños recortes en los elementos horizontales de madera de los paneles y viga solera para el paso de conducciones.

Las cajas octogonales y rectangulares de los puntos de luz, deben empotrarse y fijarse en los pa- neles, haciéndose los recortes necesarios en las cañas.

\subsection{INSTALACION SANITARIA}

Es convencional. La tuberia se empotra en el falso piso.

Las tuberias de agua que suben a los puntos de salida de ducha, lavabo, inodoro, lavadero de cocina y de ropa, van empotradas en los paneles, para conseguirlo, se recortan los travesaños de los paneles de una profundidad no mayor a 3/4".

Las tuberias de ventilación se las sube por las esquinas de las habitaciones.

\subsection{COLOCACION DE TIRAS DE MADERA EN VANOS DE PUERTAS Y VENTANAS}

En el presente sistema constructivo, los soportes de los paneles reemplazan a los marcos de puertas y ventanas; siendo necesario complementarlos con tiras de madera de $20 \mathrm{~mm}$ de espesor colocadas en sitio, para conformar los topes de las hojas de puertas y ventanas.

Igualmente se colocan tiras de madera de $20 \mathrm{~mm}$ de ancho por $20 \mathrm{~mm}$ de espesor enmarcando todos los vanos, tanto exterior como interiormente. Estas tiras sirven como limite de espesor y guía del enlucido final del muro.

\subsection{REVOQUE DE PAREDES}

Esta operación se debe iniciar cuando todos los elementos que para su fijación se necesita producir impacto, se encuentren debidamente colocados.

El revoque se aplica en dos capas como minimo, cubriéndose todas las superficies de madera de los paneles. Para conseguir adherencia del revoque a la madera, se fija en la superficie de ella, alambre negro o galvanizado \# 16 ó \# 18 en zig zag o en forma de malla.

Se puede emplear ventajosamente también con este fin, tapas metálicas de cerveza o soda clavadas a la madera.

Todas las líneas de unión de panel con panel; panel con viga solera deben ser acanaladas, para orientar la fisuración.

El revoque de las superficies de los paneles que van a estar sometidos a constante humedad, deben efectuarse integramente con mortero de cemento. 


\subsection{PREPARACION Y APLICACION DE BARRO SOBRE LA ESTERILLA DE CAÑA DEL TECHO}

Para la preparación de este barro cuya aplicación tiene por finalidad proporcionar aislamiento térmico a la edificación, se emplea tierra vegetal mezclada con paja picada. La paja aligera el peso del barro, disminuye su coeficiente de conductividad térmica y controla su fisuración por contracción de secado.

La torta de barro tendrá un espesor de $3 \mathrm{~cm}$ a $4 \mathrm{~cm}$.

Para zonas no lluviosas o poco lluviosas, el barro puede ser mezclado con asfalto de caminos RC-250 en la proporción 20:1 en volumen.

\subsection{COLOCACION DE MATERIAL DE COBER- TURA IMPERMEABLE}

En zonas de alta precipitación pluvial, es necesario colocar este tipo de cubierta, para evitar la erosión de la torta de barro por la lluvia. Esta cubierta puede estar constituida por planchas onduladas de asbesto cemento o cinc acanalado, fijadas a la estructura del techo o a la esterilla de caña.

\subsection{ENLUCIDO DEL CIELO RASO INCLINADO}

Para proteger la esterilla de caña, asi como para aumentar el aislamiento térmico, impedir que sirva de refugio de insectos y conseguir una buena apariencia, se debe revocar la superficie inferior del techo, con materiales adecuados, como mortero cemento-arena, yeso, etc. El espesor total del revoque colocado en dos etapas, debe ser aproximadamente $2 \mathrm{~cm}$.

\subsection{PREPARACION Y COLOCACION DE PUER- TAS $Y$ VENTANAS}

Se puede utilizar puertas de madera y ventanas de madera o metálicas, con vidrios o mallas plásticas o metálicas tipo mosquitero; esta última solución es recomendable para climas cálidos.

\subsection{ACABADOS FINALES}

Los acabados finales de la edificación pueden ser convencionales, tales como los materiales de piso; imprimación y pintado de paredes, enchape con mayólica de superficies húmedas de baño y cocina; barniz o pintura sobre superficies de madera vistas; cerrajeria en puertas y ventanas, etc.

\section{CONCLUSIONES}

Se puede considerar al Sistema "Quincha Prefabricada" como un sistema de madera, ya que su estabilidad estructural está confiada a elementos de este material; muy bien secundado por otro material orgánico como la caña.

Siguiendo el procedimiento constructivo explicado es posible edificar una vivienda de buenas condiciones de habitabilidad; con un coeficiente de transmitancia térmica $U=1.5 \mathrm{~K} \mathrm{cal} / \mathrm{m} 2 \mathrm{~h}{ }^{\circ} \mathrm{C}$, tanto de las paredes como de los techos, muy semejante a una edificación de muros de arcilla cocida y techo aligerado.

El aislamiento acústico, es igualmente muy semejante a este tipo de edificación.

Por otro lado, la estabilidad estructural de una edificación de quincha es óptima por ser liviana, rígida y elástica, con gran capacidad de absorción de energía sísmica, ofreciendo una muy buena alternativa constructiva en terrenos blandos, de baja capacidad portante.

Según ensayos de carga efectuados en módulos de "Quincha Prefabricada" el inicio de fisuración del revoque se presentó con una carga horizontal equivalente al producido por un sismo de una aceleración igual a $1.0 \mathrm{~g}$.

Esto nos da un indice del buen comportamiento que tienen las edificaciones de quincha prefabricada ante los mismos.

Sobre la durabilidad se puede afirmar que edificaciones coloniales de quincha, en Lima - Perú, todavía están sirviendo como viviendas o destinadas a otros usos.

Con este mismo Sistema, es posible también construir cualquier tipo de edificación, para lo cual se hará el diseño estructural correspondiente para determinar las dimensiones de los componentes.

\section{BIBLIOGRAFIA}

1. Instituto Nacional de Investigación y Normalización de la Vivienda "Proyecto de Investigación Uso de la Quincha en Edificación de Viviendas".

2. Junta del Acuerdo de Cartagena PADT-REFORT «Manual de Diseño para Maderas del Grupo Andino".

3. S. Cytryn - Centro Regional de Ayuda Técnica- Agencia para el Desarrollo Internacional (A.I.D.) "Construcción con Tierra".

4. Cuadra Carlos. "Investigaciones con Paneles de Quincha Prefabricada". Tesis de Ingenieria Civil UNI. Lima, 1983.

5. Kuroiwa J. a A. Díaz. "Viviendas Sismo-resistentes en Base a Paneles Prefabricados de Quincha». Resumen. Seminario Latinoamericano de Construcciones con Tierra en Areas Sismicas. Lima, 1983.

6. Vásquez Nelson. "Investigación sobre Construcción con Paneles de Quincha Armada". Tesis de Ingenieria Civil. UNI. Lima, 1972

7. Pontificia Universidad Católica del Perú. "Informe Final. Sistema Modular de Quincha con Suelo-Cementado".

8. Centro Interamericano de Vivienda. "El Bambú como Material de Construcción". 